DOI: 10.14526/2070-4798-2020-15-3-29-34

\title{
Correct breathing in water as the main component of teaching swimming children with mental disorders
}

\author{
Popovich N.V. ${ }^{1}$, Raspopova E.A. ${ }^{2 *}$ \\ ${ }^{1}$ Water kinds of sport Federation for people with mental disorders \\ Moscow, Russia \\ ORCID: oooo-0oo2-5767-591X,gmedian@yandex.ru \\ Russian State University of Physical Culture, Sport, Youth and Tourism \\ Moscow, Russia \\ ORCID: oooo-ooo2-1129-4492, raspopova48@mail.ru*
}

\begin{abstract}
It is known that under the influence of an active muscle activity psycho-emotional state of an organism improves, the level of anxiety decreases and this effect is especially distinct during swimming lessons, where environment is different. This environment, as special research works mention, is the means of psychic over-tensions prevention. In the opinion of many specialists, adaptive physical culture and sport is more important for a person with disabilities, than for healthy people. In this connection for children with mental disorders swimming is an effective means of physical, psychic and emotional development. The ability to control breathing is an important index of a child's behavior, it develops self-control and attention focusing. Material. The article is about the question of teaching correct breathing in water among children with mental disorders, who go in for swimming, as the main component of teaching swimming. Result. We present the exercises, which are used on land and in water for teaching correct breathing and the results of swimming speed among children with mental disorders at the beginning and at the end of the experiment, which prove the necessity to organize the directed formation of correct breathing skills in teaching swimming children with mental disorders. Conclusion. The presented methodology using special exercises for teaching breathing on land and in water among children with mental disorders helped to decrease time of 25 meters distance overcoming with free style in comparison with children from the control group. We mention lower HR after 25 meters distance overcoming, than in the control group, which shows more effective way of distance overcoming. Further it would help to teach children swimming more effectively and train them for competitions of different level.
\end{abstract}

Keywords: children with mental disorders, swimming, correct breathing.

For quotation: Popovich N.V., Raspopova E.A.* Correct breathing in water as the main component of teaching swimming children with mental disorders. Journal of Physical Education and Sport. 2020; 15(3): 23-27. DOI: 10.14526/2070-4798-2020-15-3-29-34

It is a well-known fact that swimming is a vital skill, connected with adaptation in water and the skill to move in it. Swimming lessons improve the apparatus of external respiration, form correct rhythm of breathing. [1]

It is known that under the influence of an active muscle activity psycho-emotional state of an organism improves, the level of anxiety decreases and this effect is especially distinct during swimming lessons, where environment is different. This environment, as special research works mention, is the means of psychic over-tensions prevention [3]. In the opinion of many specialists, adaptive physical culture and sport is more important for a person with disabilities, than for healthy people [2]. In this connection for children with mental disorders

swimming is an effective means of physical, psychic and emotional development. The ability to control breathing is an important index of a child's behavior, it develops self-control and attention focusing.

For children with mental disorders correct breathing in water is difficult. Many children, who have health disorders, can't control breathing and it makes teaching swimming difficult.

It is no coincidence that, in the works of neuropsychologists $[4,5,6]$ great attention is paid to the exercises, directed toward breathing control. The exercises for breathing control improve the ability of disabled children to adapt and relax in water, provide correct skill of swimming formation.

Correct breathing during swimming provides swimmers' speed of movement at the 
distance and defines the distance, which a swimmer can overcome. Tiredness and general state depend on breathing. Incorrect breathing is a serious obstacle during the training lessons, which leads to incorrect consequences. Having mastered breathing during swimming an athlete starts training lessons in water. His movement realization depends on this. The technique of correct breathing in swimming is an important moment in training a swimmer.

Success in teaching swimming depends on correct breathing organization, regardless of the way of swimming. If a person wants to learn to swim, first of all, he learns to breathe correctly. A swimmer, who had mastered correct breathing, would master the technique of any way of swimming $[7,8,9]$.

For children, who have mental disorders, teaching correct breathing is especially important. For this purpose it is necessary to use different preparatory exercises. Correct technique of breathing during swimming would help an athlete - beginner achieve the desired results quicker.

The aim of the research - correct breathing formation during front crawl swimming among children with mental disorders.

The objectives of the research:

1. To create special exercises for teaching the technique of correct breathing during front crawl swimming in children with mental disorders.

2. To define the influence of correct breathing on HR change during 25 meters distance overcoming with the help of front crawl.

3. To define the influence of correct technique of breathing on the result of swimming

Table 1 - The average results of time of 25 meters distance overcoming with free style and pulse indices within 30 seconds in children with mental disorders (experimental and control groups) at the beginning of the experiment

\begin{tabular}{|c|c|c|c|}
\hline \multirow{2}{*}{ Groups of respondents } & $\begin{array}{c}\text { Pulse within 30 } \\
\text { seconds before } \\
\text { distance overcoming }\end{array}$ & $\begin{array}{c}\text { Time of 25 meters } \\
\text { distance overcoming } \\
\text { (in sec.) }\end{array}$ & $\begin{array}{c}\text { Pulse within 30 } \\
\text { seconds after } \\
\text { distance overcoming }\end{array}$ \\
\cline { 2 - 4 } & $\mathrm{X} \pm \sigma$ & $\mathrm{X} \pm \sigma$ & $\mathrm{X} \pm \sigma$ \\
\hline Experimental group & $40,8 \pm 0,84$ & $0: 37,16 \pm 1,02$ & $80,2 \pm 0,87$ \\
\hline Control group & $40,6 \pm 0,57$ & $0: 37,8 \pm 1,7$ & $81,1 \pm 0,37$ \\
\hline \multicolumn{1}{c|}{$\mathrm{p}$} & $>0,05$ & $>0,05$ & $>0,05$ \\
\hline
\end{tabular}


The experiment was held during 3 months. The experimental group additionally fulfilled special exercises for correct technique of breathing formation for children with mental disorders on land and in water.

It should be noted that for children with mental disorders any technique and the definite actions remembering is not very easy. It means they not always understand what is wanted from them and can't fulfill the exercise correctly at once. Such children need individual approach, which is seen in an individual selection of exercises and the volume of their use. Correct breathing mastering is not an easy task. Beginners would have to control their breathing all the time. Further correct breathing during swimming would become a habit and everything would happen automatically.

Correct coordination of breathing with swimming movements considerably increase the speed of movement in water, and for children, who have difficulties with own movements coordination not only in water, but also on land, it is a special achievement.

While fulfilling the exercises on land, directed toward correct breathing study with front crawl, special attention was paid to the fact that inhalation should be fulfilled quickly and abruptly. It was done in order to breathe enough air within a short time period.

On land movements for hands movements, head turn correspondence were studied. First only one side movements, then another side movements. The coach together with children fulfilled the exercises, correcting their movements with hands. Movements were practiced until they become second nature. Then, when the movements were mastered, breathing was included. Inhalation was fulfilled when the stroke was finished during the turn of the head towards the lowered (working) hand. Special attention was paid to the turn of the head. The coach shows the order of movements using voice commands (stroke, turn, inhalation, turn, exhalation, stroke).

The stress is on the head turn, inhalation and exhalation are mastered first on land. In order to learn to control breathing, first of all it is important to fulfill the exercises first on land, then the same in water. It is directed toward training lungs and adaptation of an organism to the changing amount of oxygen.

In water the exercises were both held in static (hyperventilation) and in dynamics (movingcontrolled inhalations and exhalations). An important component of the methodology, directed toward breathing and movement correspondence, is hyperventilation. This exercise teaches to gather maximal amount of air into lungs, more than in case of ordinary inhalation. It includes the series of often deep slow inhalations and short quick exhalations. This exercise suits children with mental disorders, as they can control the depth and tempo of inhalation and exhalation. This exercise is obligatory. In water special attention was paid to the fact that inhalation should be fulfilled with the mouth over the water and exhalation under water with the mouth and nose. Exhalation starts with the nose and continues with the nose and mouth simultaneously. It helps to avoid water entrance into nasal cavity. The air is exhaled quicker with the mouth. Exhalation is stronger, than out of water, it is made with the whole lungs. It should be smooth and even and at the moment of inhalation it should release the lungs. Exhalation starts at the moment, when the head of a swimmer is under the water and finishes at the end of the stroke. If the exhalation is not complete, further inhalation would be incomplete and an athlete would feel the lack of air during the next cycle of movement during swimming.

It is necessary to follow that a child doesn't hold the breath. After inhalation it is necessary to start exhalation, it would allow to get rid of carbon dioxide. If it accumulates a swimmer would feel tiredness and even headache.

For an inhalation only face is raised from the water. The top of the head is under water. Head shouldn't be above the water, it should be even, during the exhalation the face is turned down.

During crawl it is important to have clear hands movement, breathing should correspond with it. Inhalation is made, when one hand finishes movement, the second hand gets ready to repeat it. As hands and the head work automatically we stress breathing. Breathing should be even, calm, deep and 
rhythmical. For an inhalation an athlete turns his face to the hand, which makes an active movement. It starts, when the hand raises from the water and lasts $0,3-0,5$ seconds. The moment of inhalation finishes, when the hand starts its movement - the head takes its initial position. Exhalation starts without a pause, when the face is directed toward the bottom of the pool. This is the main difficulty of hands and head work coordination. At the same time, a child shouldn't forget to make an inhalation, not only coordinate hands and head movements. The coach, being in water, turns a child's head for a breath, helping him to remember the order and coordination of hands and head movements. He also gives a signal touching the cheek of a child, that exhalation should be made. It should be noted that all exercises are fulfilled both on land and in water under direct control of a coach.

Additional examination was held at the end of the experiment after 3 months. We estimated time of 25 meters distance overcoming with free style and the frequency of pulse before and after the distance overcoming by children with mental disorders (experimental and control groups). The results are presented in table 2.

Table 2 - The average results of 25 meters distance overcoming with free style and pulse indices of the experimental and control groups at the end of the experiment

\begin{tabular}{|c|c|c|c|}
\hline Group of respondents & $\begin{array}{c}\text { Pulse within 30 } \\
\text { seconds before } \\
\text { distance overcoming }\end{array}$ & $\begin{array}{c}\text { Time of 25 meters } \\
\text { distance overcoming } \\
\text { (in sec.) }\end{array}$ & $\begin{array}{c}\text { Pulse within 30 } \\
\text { seconds after } \\
\text { distance overcoming }\end{array}$ \\
\cline { 2 - 4 } & $\mathrm{X} \pm \sigma$ & $\mathrm{X} \pm \sigma$ & $\mathrm{X} \pm \sigma$ \\
\hline Experimental group & $40,6 \pm 0,87$ & $0: 35,16 \pm 1,02$ & $75,1 \pm 0,87$ \\
\hline Control group & $40,6 \pm 0,87$ & $0: 37,2 \pm 1,7$ & $86,1 \pm 0,79$ \\
\hline $\mathrm{p}$ & $>0,05$ & $<0,05$ & $<0,05$ \\
\hline
\end{tabular}

As it is seen from the results, presented in table 2, after the experiment the time of 25 meters distance overcoming in children from the experimental group, who trained according to created methodology, considerably decreased in comparison with the children from the control group. Lower HR is also defined after 25 meters distance overcoming, in comparison with the control group, which shows more effective way of distance overcoming. Further it would help to teach children swimming and get them ready for the competitions more effectively.

\section{CONCLUSION}

On the basis of the fulfilled by us research we come to the following conclusion:

1. Children with mental disorders from the experimental group started to control breathing during swimming better, than children from the control group.

2. Special exercises use for teaching breathing on land and in water in children with mental disorders from the experimental group helped to decrease the time of 25 meters distance overcoming, in comparison with the children from the control group.

3. The results of the experiment showed that during 25 meters distance overcoming with crawl by children with mental disorders heart rate in the experimental group became lower in the average for 20 beats per minute in comparison with heart rate of children from the control group. It proves more effective energy use.

\section{REFERENCES}

1. Bulgakova N.Zh., Morozov S.N., Popov O.I. and others Health-improving, therapeutic and adaptive swimming: manual for students of higher educational establishments. Moscow: Publishing center "Academy". 2005: 432. [In Russ.].

2. Dmitriev A.K. Swimming: manual for students of higher educational establishments. Moscow: Publishing house "Physical culture and Sport". 1966. [In Russ.].

3. Fetisov A.M., Saykin S.V. Swimming in complex rehabilitation and socialization of disabled children. Science and health. 2014; 9(12). [In Russ.]. 
4. Akhutinova T.V. Methods of neuropsycological examination of 6-9 year-old children. Moscow: Publishing house V.Sekachev. 2016: 280. [In Russ.].

5. Semenovich A.V. Neuropsycological correction in childhood. Method of the substituting ontogenesis: manual. Moscow: Genesis. 2013: 474 .

6. Salikhzyanova L.I. Method of neuropsycological correction of disabled children. Modern psychology: materials of the II International scientific conference (Perm, July, 2014). Perm: Mercury. 2014: 56. [In Russ.].

7. Raspopova E.A. A coach is a teacher educator. Russian Journal of Physical Education and Sport. 2018; 13 (3): 175-179. DOI: 10.14526 / 2070-4798-2018-13-3-175-179. [In Russ., In
Engl.].

8. Paul Downward, Kirstin Hallmann, Tim Pawlowski. Assessing parental on the sports participation of children: A socio-economic analysis of the UK. European Journal of Sport Science. 2014; 14(1): 84-90. URL: http://dx.doi. org/10.1080/17461391.2013.805250.

9. Clemens Drenowatz, Sussane Kobel, Sarah Kettner, Dorothea Kesztyus, Jurgen M. Steinacker. Interaction of sedentary behavior, sports participation and fitness with weight status in elementary school. European Journal of Sport Science. 2014; 14(1): 100-105. URL: http://dx.doi.org/10.1080/17461391.2012.732 $\underline{615}$.

\section{Submitted: 28.07.2020}

\section{Author's information:}

Popovich Natalia Vyacheslavovna - director; Federation of water kinds of sport for people with mental disorders, 105318, Russia, Moscow, Ibragimova str., 32, e-mail: gmedian@yandex.ru

Raspopova Eugenia Andreevna - doctor of pedagogics, professor; Russian State University of Physical Culture, Sport, Youth and Tourism, 105122, Russia, Moscow, Sirenevyi aven., House 4, e-mail: raspopova48@mail.ru 